\title{
BMJ Open Quality Ward round template: enhancing patient safety on ward rounds
}

Niall Gilliland, Natalie Catherwood, Shaouyn Chen, Peter Browne, Jacob Wilson, Helena Burden

To cite: Gilliland N, Catherwood N, Chen S, et al. Ward round template: enhancing patient safety on ward rounds. BMJ Open Quality 2018;7:e000170. doi:10.1136/ bmjoq-2017-000170

Received 20 July 2017 Revised 12 March 2018 Accepted 8 April 2018

\section{Check for updates}

Department of Urology, Southmead Hospital, The Severn Deanery, Bristol, UK

Correspondence to Mr Niall Gilliland, Department of Urology, Southmead Hospital, The Severn Deanery, Bristol, UK; niallgilliland@nhs.net

\begin{abstract}
Introduction and aims Concerns had been raised at clinical governance regarding the safety of our inpatient ward rounds with particular reference to: documentation of clinical observations and National Early Warning Score (NEWS), compliance with Trust guidance for venous thromboembolism (VTE) risk assessment, antibiotic stewardship, palliative care and treatment escalation plans (TEP). This quality improvement project was conceived to ensure these parameters were considered and documented during the ward round, thereby improving patient care and safety. These parameters were based on Trust patient safety guidance and CQUIN targets. Method The quality improvement technique of plando-study-act (PDSA) was used in this project. We retrospectively reviewed ward round entries to record baseline measurements, based on the above described parameters, prior to making any changes. Following this, the change applied was the introduction of a ward round template to include the highlighted important baseline parameters. Monthly PDSA cycles are performed, and baseline measurements are re-examined, then relevant changes were made to the ward round template.

Summary of results Documentation of baseline measurements was poor prior to introduction of the ward round template; this improved significantly following introduction of a standardised ward round template. Following three cycles, documentation of VTE risk assessments increased from $14 \%$ to $92 \%$. Antibiotic stewardship documentation went from $0 \%$ to $100 \%$. Use of the TEP form went from $29 \%$ to $78 \%$.

Conclusions Following introduction of the ward round template, compliance improved significantly in all safety parameters. Important safety measures being discussed on ward rounds will lead to enhanced patient safety and will improve compliance to Trust guidance and comissioning for quality and innovation (CQUIN) targets. Ongoing change implementation will focus on improving compliance with usage of the template on all urology ward rounds.
\end{abstract}

\section{INTRODUCTION}

This quality improvement project took place across the urology department of a large tertiary referral centre, with daily ward rounds.

It is well known that surgical ward rounds are held at a fast pace, and within urology, this is no different. Due to the speed of surgical ward rounds, it is not uncommon for there to be discrepancies in the documentation of the information given to patients and their management plans. ${ }^{1}$ It was highlighted within clinical governance meetings that some important pieces of information could be missed, and this could impact on patient safety. The areas that were highlighted included: documentation of clinical observations and National Early Warning Score (NEWS), compliance with Trust guidance for venous thromboembolism (VTE) risk assessment documentation, antibiotic stewardship, end-of-life care and treatment escalation plans (TEP). There was no set format for junior doctors to follow to ensure all of these pieces of information were documented.

Many hospitals use a ward round proforma to ensure important clinical information is documented, ${ }^{2}$ and in intensive care, ward round template sheets have been used for some time with excellent results and improvements in patient safety. ${ }^{34}$

The ward round is an important part of the day, and the documentation provides a record of the treatment so far and the plan going forward. It is also a medicolegal record $^{5}$; therefore, the content and clarity of the record is of the utmost importance. ${ }^{6}$

\section{AIMS}

To improve patient safety, compliance to CQUIN targets, and ward round documentation, a standardised paper-based ward round template was created. The ward round template would include important patient safety parameters, as decided by Trust and National guidance, and could easily be added into the patient's notes.

\section{MEASURES}

Baseline measures were decided based on Trust and national patient safety guidance; these were: clinical observations, NEWS, VTE risk assessment, antibiotics, and palliative care or treatment escalation. Documentation in ward round entries were reviewed, and information about documentation of baseline 


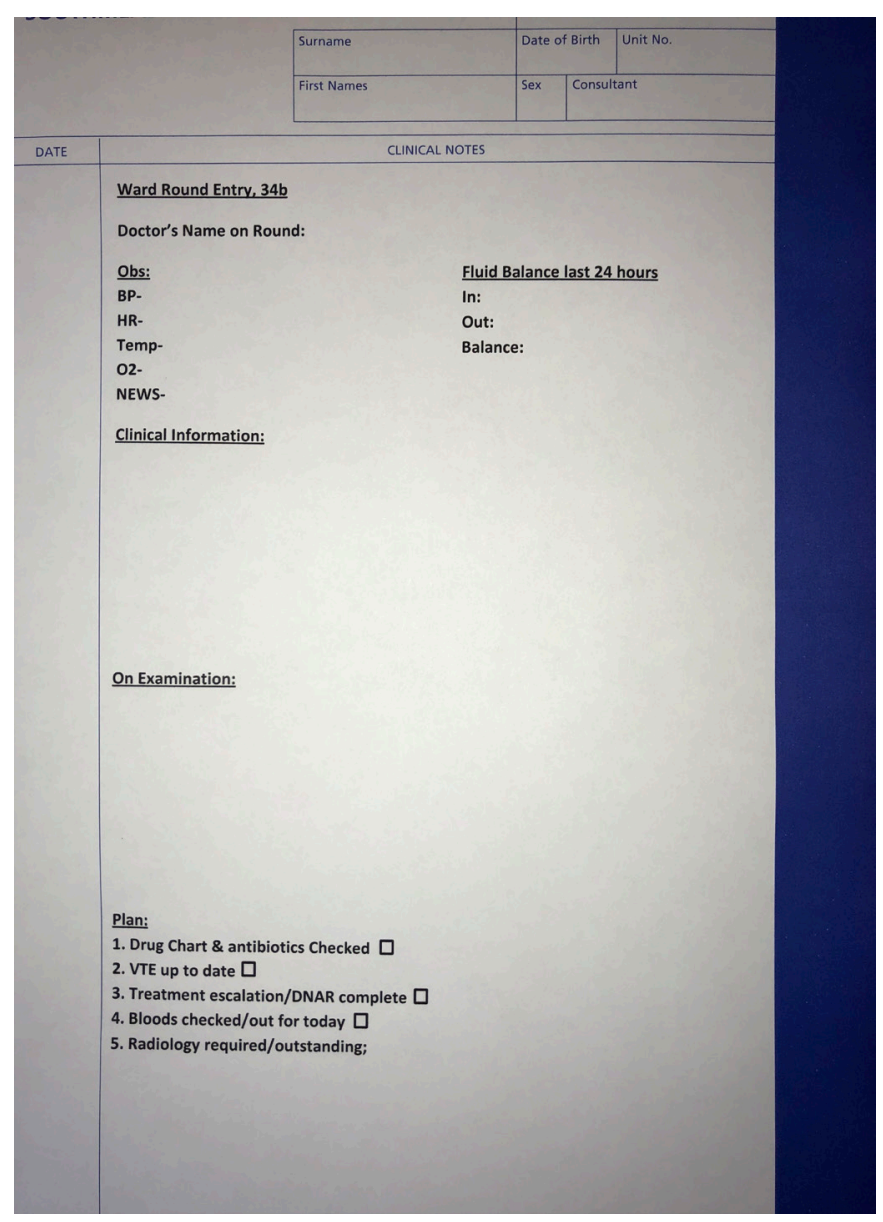

Figure 1 Version one of the ward round template shown on continuation paper.

measures was recorded as present or absent. In regard to VTE risk assessment, this also needed to be recorded on Lorenzo, which is a patient information management system. An initial review of baseline measures was carried out prior to change implementation, then the change was implemented and repeated plan-do-study-act (PDSA) cycles were completed.

\section{CHANGES}

A ward round template was devised after the initial data were collected based around the baseline parameters identified. After each PSDA cycle was completed, the ward round template was changed based on the results. Each PDSA cycle will be described in more detail below.

\section{PDSA cycle 1}

\section{Plan}

Based on the discussions at clinical governance, a robust system to record important information and discussions on the daily ward round was needed. The baseline measures that were seen as essential to document were: clinical observations (including NEWS), VTE risk assessment, antibiotics and palliative care/treatment escalation decision. These were chosen based on Trust and national patient safety guidelines. The requirement was

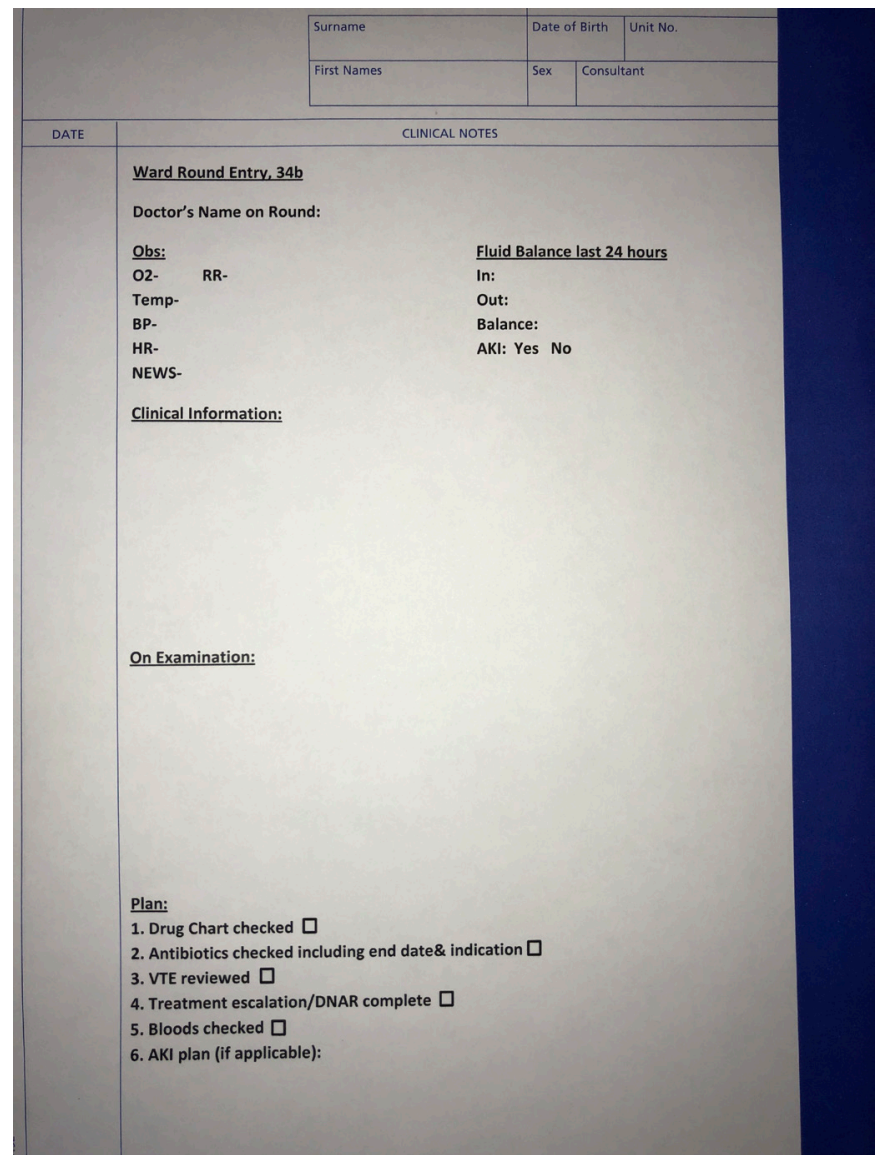

Figure 2 Version 2 of the ward round template shown on continuation paper.

to develop a paper-based template that could be printed onto continuation paper and entered into the patient notes. It needed to be simple to follow and to save time when filling in the ward round entry for that day. The template was developed by a urology core trainee (CT) in conjunction with the urology foundation year one (F1), and general practitioner (GP) trainee doctors; it was shown to the consultant that was supervising the project for approval before using (figure 1).

\section{Do}

Version 1 of the template was used on a daily basis for all of the urology inpatients for 1 month.

\section{Study}

A day was chosen at random after 1 month of using the template, and the level of compliance to using the sheet and the documentation of the baseline measures were recorded. This was compared with the data collected prior to using the template to see if there had been an improvement. Verbal feedback was taken from the urology doctors (from F1 to registrar), nurses and pharmacists who were using the templates in order to make changes to improve the layout or to add any extra points in. 


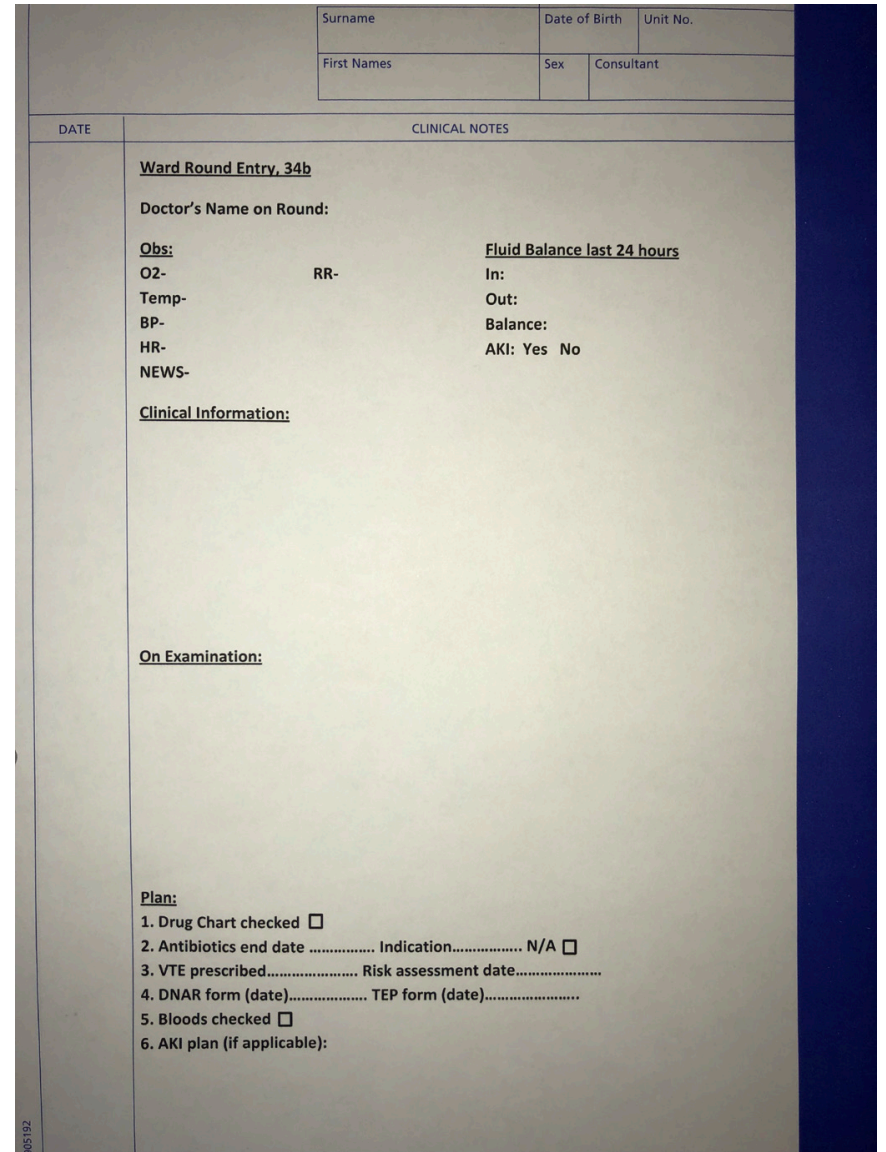

Figure 3 Version 3 of the ward round template shown on continuation paper.

\section{Act}

The template was well received and was being used for the majority of urology patients. An improvement in documentation had been seen within all of the baseline measures. Several areas for change were highlighted, and these will be discussed in PDSA cycle 2 .

\section{PDSA cycle 2}

Plan

Discussions with the junior doctors and pharmacists highlighted that there was no prompt in version 1 of the template to discuss acute kidney injury (AKI). Within urology, it is common to have patients with an AKI, and the prompt identification of this and implementation of a plan to treat the AKI are important to prevent the morbidity associated with it. It is a mandatory section within the Southmead Hospital discharge summary to discuss any AKI, and how it was treated, so making it clear if, and when this had happened, helped the doctor comple this section of the discharge summary. The pharmacists had also highlighted that using a tick box to show that antibiotics had been discussed was useful, but documenting the antibiotic, duration of treatment and indication would be more useful as it should reflect what was documented in the drug chart and any discrepancies would be easier to pick up. The nurses had highlighted that VTE risk assessments were being checked, but the documentation around the decision was still unclear (especially if any aspect of it was being withheld).

Do

Version 2 (figure 2) of the template was rolled out for use on the ward rounds; the tick boxes had been amended to reflect the changes mentioned above.

\section{Study}

A day was chosen at random 1 month after the introduction of the updated template, and the level of compliance of using the template and the documentation of the baseline measures were recorded. The sheet continued to be used regularly, and documentation of the baseline measures improved further. Feedback continued to be positive from all members of the multidisciplinary team, but suggestions were proposed to improve the template further.

\section{Act}

Based on feedback, in some aspects of the template, it was felt a tick box did not give enough documentation. For example, when documenting antibiotic discussions, the pharmacists requested that the antibiotic indication and duration is written and that it matches the drug chart. The palliative care team also asked for more information around treatment escalation discussions.

\section{PDSA cycle 3}

Plan

Responding to feedback white space was introduced to the antibiotics section of the plan, so doctors could fill in the antibiotic, indication and duration of treatment. White space was added to the VTE risk assessment section to document that it was prescribed and that the risk assessment was up to date. There was white space added to the do not resuscitate (DNAR)/TEP section, this was so a date could be highlighted of when the discussion took place; this date could be searched in the notes and cross-referenced with the DNAR/TEP form at the front of the patient folder. The introduction of white space was to encourage improved communication through descriptive writing as opposed to just ticking a box. Due to the success of the form, it was included in the enhanced recovery booklet where all documentation is kept for patients on the enhanced recovery programme.

\section{Do}

Version 3 (figure 3) of the new template was rolled out for use on the ward and in the enhanced recovery booklet, the template was amended to reflect the changes above.

\section{Study}

A day was chosen at random 1 month after the introduction of the updated template, and the level of compliance of using the template and the documentation of the baseline measures were recorded. The template was being used more regularly, and the documentation of the baseline measures continued to improve and was 


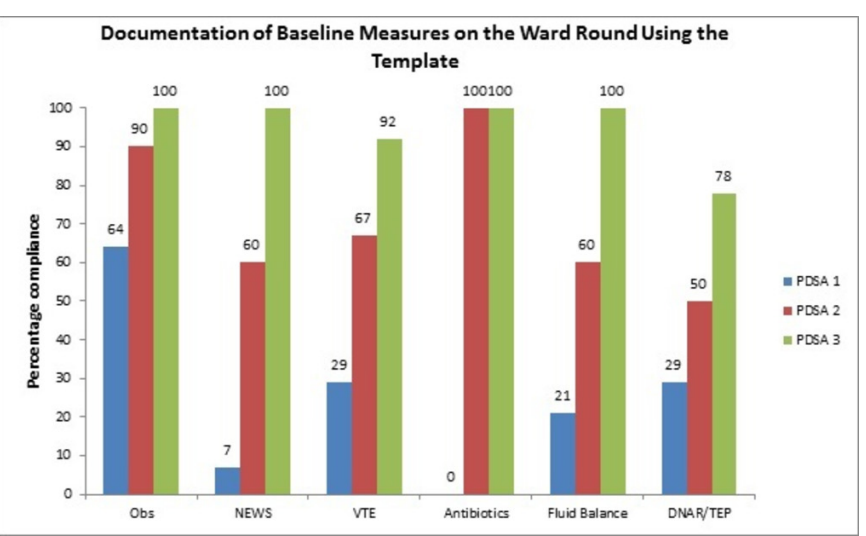

Figure 4 Graph showing the percentage compliance of documentation of the baseline measures on the ward round using the template for each PSDA cycle. PDSA, plan-dostudy-act; TEP, treatment escalation plan.

$100 \%$ in some cases. Feedback from the multidisciplinary team continued to be good, and minor suggestions for improvement were being raised.

Act

As the documentation of baseline measures had improved significantly, and only minor suggestions were being made to improve the template, we decided that no further PDSA cycles were required, unless a significant change to the template was suggested. Minor changes could be made by the junior doctors on the ward to suit their needs. One year after the creation of the ward round template, it was still being used on the ward and continued to receive good feedback from the multidisciplinary team.

Figures 1-3 show how the ward template evolved.

\section{METHODS}

Ward round entries of all elective urology patients were reviewed retrospectively in each PDSA cycle; data were recorded on 1 day at random 1 month after the changes had been made. Data were recorded and analysed on a Microsoft Excel spreadsheet, and the information was recorded as present or absent. In respect to VTE risk assessments, they needed to be recorded in the notes and electronically, as the computer programme (Lorenzo) was where the Trust complied their reports on VTE risk assessment compliance from.

\section{RESULTS}

Initial compliance to all of the areas that were highlighted was poor (see figure 3, PDSA cycle 1), clinical observations were recorded in $64 \%$ of patients, but for all the other areas of clinical information, baseline parameters were recorded less than $30 \%$ of the time. After introduction of the ward round template, compliance in all areas increased to $>50 \%$ after PDSA cycle 2 , and to $>75 \%$ after PDSA cycle 3 . In many areas, compliance was $>90 \%$ by the end of this period of study.

Figure 4 shows how compliance increased over time.
In PDSA cycle 1, 14 patient's notes were analysed.

In PDSA cycle 2, 17 patient's notes were analysed, and the template was used $60 \%$ of the time.

In PDSA cycle 3, 14 patient's notes were analysed, and the template was used $65 \%$ of the time.

\section{DISCUSSION}

The airline industry use checklists every day to improve safety, and this has been replicated in healthcare settings by the introduction of checklists into daily clinical practice. A good example of this is the WHO checklist prior to surgery, and the positive effect that this has had to reduce postopoperations complications and deaths. ${ }^{7}$

This quality improvement project has highlighted the usefulness of a simple ward round template to ensure that important clinical information is recorded, which can help improve patient safety and communication on the ward.

The ward round sheet was used $60 \%$ and $65 \%$ of the time in cycles 2 and 3, respectively. While our urology patients are mainly based on a specific urology ward, emergency patients are often outliers, and therefore there could be a lack of availability of ward round templates on other wards, which could partially explain the why sheets were not used all of the time. Another explanation could be the juniors not having time to print out copies prior to the ward round.

Feedback from the junior doctors showed that they liked to use the template. They felt that it was a good aide memoir while on ward rounds and also empowered them to challenge seniors for more information about that patient or to ensure important documentation was complete, like VTE risk assessments or treatment escalation plans. This meant that they were keen to use the template and had bought into the concept. The template was still being used on the ward and in the enhanced recovery booklet 1 year after its conception. This was after the doctor who created the template had left the department, which demonstrates the value that the template brought to the department.

Feedback from the nursing team was that the ward round entries were set out in a logical fashion, and they could understand the plans and jobs that needed to be done for that day.

Clinical outcomes were not recorded as part of this project, but focusing on areas like clinical observations, VTE risk assessment and antibiotic stewardship will clearly have a positive effect on safer clinical outcomes for patients. By clearly documenting clinical observations, a deteriorating patient can be highlighted to a senior colleague, and an appropriate management plan can be initiated swiftly. Anticoagulation decisions are not always straightforward in urology where we see lots of patients with haematuria, who may already be on anticoagulation, or have comorbidities making them high risk for thrombosis. Reasons for and against anticoagulation were clearly documented, making nurses more confident to give or not give anticoagulation to patients. We could also ensure patients were on the correct antibiotics for the correct 
duration, therefore reducing the risk of antibiotics resistance and Clostridium difficile infection. Focusing on these areas made patient's stay in hospital safer and helped with timely, safe discharges.

Limitations of this project include low numbers of patients on each day; the average number of patients was 15. Trends can be identified, but higher number would be needed to draw firm conclusions; unfortunately, the number of urology patients admitted was not something we could control. Prospective data capture when recording what was said and what was documented on ward rounds prior to creating the template, and during each PDSA cycle, may have identified any breakdown of communication and may have enabled us to improve the template further. We also did not look at patient outcomes; an assumption was made that, for example, if the VTE risk assessment was documented, then there would be less incidences of deep vein thrombosis (DVT) or pulmonary embolism (PE) in hospital.

\section{CONCLUSIONS}

The introduction of a ward round template significantly improved the documentation of important pieces of clinical information that impact directly on patient safety.

Communication between doctors and nurses improved, and decisions that were made on ward rounds were fully documented in the clinical notes.

A ward round template is easy to formulate and tailor to a department's individual needs and can be changed quickly to suit the dynamic environment of any ward.

Clear documentation of key clinical information will make a patient's stay in hospital safer and ensure timely and safe discharges.

\section{RECOMIMENDATIONS}

A template should be used on ward rounds to improve patient safety, compliance to Trust guidelines and CQUIN targets.

Acknowledgements We would like to thank The Bristol Urological Institute staff on 34b Southmead Hospital.

Contributors NG developed the idea of the ward round template and is involved in modifying new versions of the template; he was involved in data collection and analysis and writing the paper. NC, SC, PB and JW were involved with data collection and analysis. HB was the project supervisor.

Funding The authors have not declared a specific grant for this research from any funding agency in the public, commercial or not-for-profit sectors.

Competing interests None declared.

Provenance and peer review Not commissioned; externally peer reviewed.

Open Access This is an Open Access article distributed in accordance with the Creative Commons Attribution Non Commercial (CC BY-NC 4.0) license, which permits others to distribute, remix, adapt, build upon this work non-commercially, and license their derivative works on different terms, provided the original work is properly cited and the use is non-commercial. See: http://creativecommons.org/ licenses/by-nc/4.0/

(c) Published by the BMJ Publishing Group Limited. For permission to use (where not already granted under a licence) please go to http://www.bmj.com/company/ products-services/rights-and-licensing/

\section{REFERENCES}

1. Fernando KJ, Siriwardena AK. Standards of documentation of the surgeon-patient consultation in current surgical practice. $\mathrm{Br} J$ Surg 2001;88:309-12.

2. Duxbury O, Hili S, Afolayan J. Using a proforma to improve standards of documentation of an orthopaedic post-take ward round. BMJ Qual Improv Rep 2013;2:u200902.w699.

3. Gawande A. The checklist manifesto: how to get things right. New York: Metropolitan Books, 2010.

4. Gawande A. The checklist: if something so simple can transform intensive care, what else can it do? 86th edn. New York: The New Yorker, 2007:86-101.

5. Mann R, Williams J. Standards in medical record keeping. Clin Med 2003;3:329-32.

6. Al-Mahrouqi H, Oumer R, Tapper R, et al. Post-acute surgical ward round proforma improves documentation. BMJ Qual Improv Rep 2013;2:u201042.w688.

7. Haynes AB, Weiser TG, Berry WR, et al. A surgical safety checklist to reduce morbidity and mortality in a global population. $N$ Engl $\mathrm{J}$ Med 2009;360:491-9. 